\title{
La polémica del romanticismo en Chile. Dos artículos desconocidos
}

UNa de las contribuciones más sólidas que aportó el erudito chileno Norberto Pinilla al estudio de la historia literaria de Chile, fué la colección de artículos que integran la célebre polémica del año 1842 sobre el romanticismo. ${ }^{1}$ Esa colección la hizo a fin de documentarse para sus dos valiosos trabajos sobre la generación literaria de aquel año. ${ }^{2} \mathrm{El}$ valor de su libro es precisamente, como lo afirmó en su prólogo, el de reunir en un solo volumen varios materiales que de otro modo serían muy difíciles de manejar, por hallarse dispersos en bibliotecas públicas y particulares - materiales, además, indispensables para el estudio de uno de los períodos más interesantes de su país y de la América Hispana.

Antes de que el catedrático chileno publicara su libro, estando yo en Chile e ignorando que él tenía la intención de publicar esos artículos coleccionados, formé mi propia colección de ellos. Hacía una década que me interesaba por el romanticismo del mundo hispánico, ${ }^{3}$ y estaba entonces en la América del Sur documentándome para varios trabajos sobre la cultura literaria de aquellos países. No pude encontrar en Santiago el artículo del argentino Vicente Fidel López, Clasicismo y romanticismo, porque -como advierte Pinilla en su prólogo-, la colección de la Revista de Valparaiso, de 1842, no está en la Biblioteca Nacional de esa capital, y se la puede consultar únicamente en bibliotecas privadas. Pero averigüé que habia. un ejemplar en la Biblioteca Widener, de la Universidad de Harvard, y más tarde pude conseguir una fotocopia del artículo que me faltaba. 
Muy poco después de que yo reuniera la mía, la colección de Pinilla salió de la imprenta. Los estudios coleccionados y publicados por el erudito chileno comprenden los dos trabajos de Vicente Fidel López que aparecieron en la Revista de Valparaiso y en la Gaceta del Comercio ; ${ }^{4}$ tres de Salvador Sanfuentes, publicados en el $\mathrm{Se}$ manario de Santiago; uno de José Joaquín Vallejo, en el Mercurio de Valparaíso; nueve artículos de Sarmiento del mismo periódico; uno de Antonio García Reyes, que salió a luz en el Semanario, y otro más, anónimo, que fué publicado en la Gaceta de Comercio.

Pero faltan en la colección de Pinilla dos artículos más que me he decidido a publicar ahora, para que la documentación de la polémica quede tan completa como sea posible.

El primero de los dos no es, en verdad, más que una corta nota, sin duda alguna de Sarmiento. Lo insertó en el Mercurio del 31 de julio, el mismo día que salió su artículo Volvamos todos a la moderación, con cuyo espíritu moderado está en perfecta armonía. No fué Pinilla el único que dejó de reimprimirlo. Tampoco figura entre los artículos coleccionados por Luis Montt para el primer tomo de las Obras de Sarmiento. ${ }^{5}$

El motivo de la nota de Sarmiento era el siguiente. En su artículo del 25 de julio, El romanticismo según el Semanario, el joven proscrito argentino había tomado la palabra coliseo por el nombre de algún escritor romano. Criticaba la necesidad en que se creían los redactores del Semanario de apoyarse en autoridades clásicas y dijo asi:

Se necesita además estar muy al corriente de los escritos de la época, del pro y del contra de las cuestiones literarias que se han ventilado en Europa; y dado el caso que crea necesatio apoyarse en autoridades, tomarlas entre los grandes hombres de la civilización moderna, que saben más cada uno de ellos, y cuyas opiniones son de más peso, que las de cualquier autor de siglos que no nos pertenecen y que ya han muerto para nosotros; y mucha mengua sería un escritor moderno, salirnos a cada paso Estacio, Coliseo, Pradon, Horacio, Moratín y otras reputaciones de an, taño, sin decirnos nunca nada de lo que hacen, dicen o piensan los escritores de nuestra época, dejándonos sospechar que en lugar de ser un literato de su siglo, es un arqueologista, o algún escapado de una época pasada...6 
A pesar de lo cómico de este error de Sarmiento, no es de extrañar que se hubiera equivocado. Coliseo en el sentido de teatro era un término nuevo, que se había introducido recientemente en Chile. ${ }^{7}$ Tampoco es de extrañar que en el calor de la controversia, Sanfuentes - cuyo artículo Sarmiento criticaba--, sacara partido del error de su adversario. Asi lo hizo en el tercer número del Semanario, en el cual publicó un divertido diálogo entre la personificación de este periódico y la del Mercurio; diálogo que contiene los siguientes párrafos:

El Merc. $-Y$ para merecer el nombre de literato, de hombre de luces, de escritor de este siglo y de escritor concienzudo, es necesario apoyarse en autoridades de los grandes hombres de la civilización moderna, y no en reputaciones de antaño como las de Estacio, Coliseo... El Sem.-Aguardad, aguardad hermano, que os habéis equivocado creyendo que Coliseo fué un autor antiguo. Coliseo es el teatro de Santiago, según la denominación que le daba en sus románticos carteles un romántico compatriota vuestro, y por eso el Semanario lo escribió en letra bastardilla. 8

Tres días después, Sarmiento publicó la nota que reproducimos abajo, en la cual confesó muy lealmente su error. Aunque sea nula la importancia de aquellos renglones para la cuestión del romanticismo, vale la pena de reproducirlos porque son un indicio más de la grandeza de aquella alma noble, cuando confiesa muy franca y sencillamente que: "Nuestro hermano menor el Semanario nos ha hecho caer en cuenta de un error que habíamos cometido, al creer al Sr. Coliseo con letra bastardilla un autor clásico: Nos había parecido que había un tal Coliseo romano, y como se hablaba de Estacio nos equivocamos."

A mi parecer, empero, la nota de Sarmiento no carece de importancia, porque está relacionada con un problema que de algún tiempo a esta parte me interesa: el de la autenticidad de ciertos artículos atribuídos a él, y reproducidos en sus Obras, que tal vez no sean suyos. ${ }^{9}$

Esta nota enfoca la atención del que lee con cuidado los artículos de Sarmiento, sobre el hecho de que éste no comprendía el nuevo y bastardo significado del término coliseo, cuando escribió su trabajo del día 25 de julio. Y por lo tanto nos induce a examinar más detenidamente otro artículo de la polémica, el del 27 de julio, 
Paréntesis formado por una correspondencia imparcial, el cual ha sido atribuído siempre a Sarmiento, pero cuyo autor emplea esa palabra en su nueva acepción, ignorada dos días antes por el joven argentino. ${ }^{10}$

Hay seis motives que, tomados en conjunto, me hacen creer que este artículo no es de Sarmiento. En primer lugar, como acabamos de ver, y esto es la clave del problema, el autor del artículo usa la palabra coliseo en el sentido de teatro ("... esta falta de discernimiento en las piezas que en el Coliseo de Santiago se han representado y que han sido aplaudidas por la sencilla razón de no haberse podido comprender"). Sarmiento, en la nota que publicamos ahora, se burla de esta acepción de la palabra, repitiendo el mismo adjetivo usado por Sanfuentes. La llama "un romántico título dado a nuestro teatro de Santiago".

$2^{\circ}$ Tiene la forma de una carta dirigida a los editores del Mercurio. De ahí el título Paréntesis formado por una correspondencia imparcial - título que le puso Luis Montt, al recopilar los materiales para el primer tomo de las obras de Sarmiento, y que Pinilla tuvo a bien conservar, aunque no aparece en el número 4162 del Mercurio. Allí, en efecto, figura en la sección "Correspondencia" (págs. 2 y 3 ).

30 En su forma original, la carta está firmada "Uno con otro", firma que no reproducen ni Montt ni Pinilla.

49 El punto de vista del autor hacia el romanticismo (o el de los autores, dada la existencia de la firma que acabamos de señalar) difiere bastante del de Sarmiento. Este, en los dos artículos anteriores, afirma que el romanticismo ha muerto ya ("Un artículo Romanticismo escrito en el año de 1842, es decir, después de diez años que la escuela romántica en Europa fué enterrada y sepultada al lado de su antecesor en literatura, el clasicismo, porque ambos son ánimas del otro mundo, que Dios bendiga ..."; ${ }^{11}$ "La crítica europea cebó su diente en las carnes del romanticismo cuando este monstruo de cien cabezas estaba lleno de vida..."; "Si el romanticismo tuvo en vida enemigos ¿qué diremos de los que salen a los diez años después a dar gritos en el aire? Diremos que estos tales tienen la suerte de andar siempre atrasados en las horas.") ${ }^{13}$ Ahora bien, para el autor del llamado Paréntesis, la escuela romántica sigue en plena vida, y recomienda a los chilenos: 'Si hay algún pueblo para 
quien el romanticismo venga mejor, es cabalmente para Chile; por consiguiente, cualquiera limitación de esta literatura, es un paso atrás, y un elemento de mal gusto." ${ }^{14}$ En lugar del epitafio que propone Sanfuentes para la lápida del sepulcro del romanticismo ${ }^{15}-$ sepulcro que no se ha erigido todavía, según la opinión de este escritor-, propone otro para una tumba que sí existe: la del clasicismo.

$5^{\circ}$ Sarmiento repite varias veces que el romanticismo ha sido substituido por otra escuela: la socialista o progresista ("la escuela socialista o progresista se ha parado sobre el pedestal firme y seguro de las necesidades de la sociedad..."; ${ }^{16}$ "... no fué el caduco e impotente clasicismo quien tuvo la gloria de darle el golpe mortal (al romanticismo); la tumba lo había reclamado hacía tiempo; fué otro campeón más joven, más ardiente y más temible; fué la escuela progresista la que se apoderó del campo de batalla y se apropió los despojos de los contendientes"). ${ }^{17} \mathrm{El}$ autor del Paréntesis no cree que otra escuela haya sobrevivido todavía, aunque prevé la posibilidad de un sucesor del romanticismo: "Si algún día sucede a esta escuela otra, no habrá por qué admirarse, porque en esto se sigue el orden natural de las cosas, que rechaza siempre lo que no es de la época; tal es la ley del progreso."

$6^{\circ} \mathrm{Al}$ proponer el susodicho epitafio del clasicismo, el autor de la carta lo escribe en verso, to cual hace particularmente dudosa la atribución a Sarmiento. Desde los tempranos días del Canto a Zonda y la resultante correspondencia con Alberdi, Sarmiento se guardó muy bien de escribir versos. ${ }^{18}$ Es muy poco probable que en medio de la calurosa polémica de 1842 se expusiera al ridículo de los jóvenes chilenos componiendo otros, ya fuesen serios o burlescos.

El segundo artículo que reproducimos abajo fué publicado en la Gaceta del Comercio del día 8 de agosto: ${ }^{19}$ un día antes de la publicación, en el mismo periódico, del último artículo recogido por Pinilla. Es muy posible que haya motivado el último trabajo anónimo de la Gaceta. Como otros artículos que integran la polémica, éste se halla en forma de carta a los editores. Por esto y por lo que dice de las ideas expuestas anteriormente en la Gaceta, se comprende que no es del autor de ellas, Vicente Fidel López.

Podría muy bien servir de conclusión a la controversia, porque analiza las pretensiones y las contribuciones de los tres periódicos que intervinieron en ella. Critica despiadadamente el papel de Sar- 
miento en la polémica, tachándole de verboso, de haber escrito sólo para demostrar sus conocimientos sobre retórica, y de haber introducido "sandeces y chocarrerías" ajenas a la cuestión; pero está de acuerdo con su análisis del Ruy Blas, de Víctor Hugo. Califica de ecléctico al Semanario y lo defiende contra la Gaceta afirmando que ésta no ha comprendido las intenciones de su adversario y que, por consiguiente, ha basado toda su intervención en premisas falsas. Pero en lo que respecta a la exposición del significado del romanticismo, da la victoria a la Gaceta, porque cree que su crítica es más razonable y sus ideas son más justas.

Publicamos a continuación los dos artículos desconocidos.

El tercer número del Semanario trae un diálogo de que apenas hacíamos reminiscencia, pero muchas de las palabras que ponen en nuestra boca son conocidamente nuestras, y es preciso creer o reventar. Nuestro hermano menor el Semanario nos ha hecho caer en cuenta de un error que habíamos cometido, al creer al Sr. Coliseo con letra bastardilla un autor clásico. Nos había parecido que había un tal Coliseo romano, y como se hablaba de Estacio nos equivocamos. Ya sabemos ahora que Coliseo no es autor, sino un romántico título dado a nuestro teatro en Santiago por un compatriota del Semanario y del Mercurio.

Pero díganos nuestro hermano el Semanario la verdad. ¿A quién ha querido dar la lección, de la palabra Coliseo; al público, a nosotros, o a sí mismo? ¿ Se propone en efecto persuadirnos que Coliseo no es autor? Vamos, se propone denunciar al público nuestra ignorancia. ¿Qué candor!

Por lo demás la conversación o diálogo entre ambos contrayentes no deja de tener su interés, y nos parece corresponder a nuestros deseos de volvernos a las buenas. Sí, es mucho mejor que nos andemos con tiento y nos riamos, antes de que nos pongamos de mal humor. Después nos volveremos a ver.

(Mercurio de Valparaíso, núm. 4166, 31 de julio de 1842). 


\section{Correspondencia}

Sres. Editores de la Gaceta:

¿Qué es romanticismo? He aqui una cuestión por la que se han devanado los sesos Mercurio, Gaceta y Semanario y de que no hemos sacado hasta ahora nada en limpio. El primero parece que se ha propuesto agotar toda su verbosidad; por este lado es verdaderamente romántico. De la lectura de sus artículos se saca muy poco o nada de provechoso; el entendimiento queda 'vacío y los oidos retiñendo con sus retumbantes frases. La Gaceta es más juiciosa; pero también cojea de otro lado; le gusta mucho llenarnos los suyos con las citas de cuantos autores conoce: ésta es su muleta. El Semanario, por fin, en su tercer número nos ha insertado dos o tres artículos que no presentan por sí nada de interesante. Pero pasemos a la cuestión.

¿Qué es romanticismo? - La independencia en literatura; definición del mișmo Víctor en ella — su Hugo- y muy conocida ya: no hay para qué detenernos. Su origen se deriva especialmente de la ignorancia de la Edad Media, en que los hombres desconociendo las reglas a que sus antepasados habían sujetado la literatura, se vieron precisados a seguir el vuelo de su imaginación. De aquí data la época del romanticismo.

Este sistema, como todos, se llevó más allá de los límites trazados por la recta razón: bajo el nombre de románticas, como dice muy bien el Semanario, se dieron a luz terribles monstruosidades; produjo más errores que el clasicismo, y era naturalísimo que fuese así, supuesto que es mucho más fácil errar sin reglas que con ellas. En Inglaterra el primero que demostró con bastante perfección las bellezas del romanticismo fué Shakespeare, ese genio inmortal que a pesar de sus rarezas y extravagancias, como dice Moratín en sus notas sobre la traducción de Hamlet, puede citarse todavía como uno de los tipos del romanticismo inglés. Pero era imposible que un sistema reǵenerador como éste dejase de tener atacadores: Pope y Addison, clásicos refinados, se empeñaron después en denigrarlo; $\mathrm{y}$ lo habrían conseguido, si no hubiese aparecido un Lord Byron y un 
Walter Scott que, por medio de sus admirables genios, consiguieron sepultar en la tumba al clasicismo. Esto es por lo que toca a la literatura inglesa: pasemos ahora a la francesa. Al principio sucedió poco más o menos lo mismo: hasta que nacieron Víctor Hugo, Dumas, Lamartine y Scribe que, después de una corta lucha con Delavigne y la memoria del clásico Delille, hicieron también triunfar al romanticismo.

Hemos bosquejado ligeramente la historia del romanticismo, para que pueda entenderse más fácilmente el juicio que hemos formado de los artículos que con este motivo han publicado el Semanario, el Mercurio y la Gaceta.

E1 sistema que parece adoptar el primero en su artículo es el eclecticismo, esto es, el justo medio entre los dos sistemas: clasicismo y romanticismo. No concebimos a la verdad, cual sea el justo medio entre dos sistemas tan contradictorios, y entre los cuales el uno de ellos no es susceptible de límites. Esperamos que en su próximo número nos explique con más claridad su sistema y entonces daremos nuestra opinión sobre el particular. Acerca de la crítica que hace del Ruy Blas de Víctor Hugo estamos en todo conformes con el Mercurio. No encontramos en esta pieza ninguna de las inverosimilitudes que el Semanario.

El Mercurio se propuso criticar el artículo "romanticismo" del Semanario. $Y$ preguntamos ahora ¿lo ha conseguido? ¿qué es 10 que ha hecho después de llenar cuatro o cinco Mercurios con sus difusos artículos? ¿que el Ruy Blas de Víctor Hugo es bueno? Con dos o tres renglones bastaba para eso. Lo que creemos es que su objeto principal fué manifestarnos sus conocimientos sobre Retórica, su buena manera de escribir. Pero si verdaderamente fué éste (lo que no dudamos) debió haberles puesto por epígrafe "conocimientos sobre Retórica" para haberles evitado su lectura a muchos que creyendo encontrar dilucidadas sobre el romanticismo algunas ideas que anteriormente había vertido en la Revista el señor López, han tenido que sufrir una pena de esperanza frustrada. También debe haberse abstenido, cualquiera que hubiese sido su objeto, de introducir sandeces y chocarrerías tan ajenas de la cuestión. Por último, le aconsejamos que procure informarse mejor del contenido (y) del espíritu de los artículos que se propone criticar y de ese modo 
no veremos críticas como las del artículo Semanario de la Recoleta Dominica del Semanario.

Vamos por fin a la Gaceta. La crítica que hace del Romanticismo es más puesta en razón; se conoce que sus ideas sobre el particular son más justas. Pero es preciso que advirtamos que toda ella rueda sobre dos premisas falsas: $1^{\circ}$ suponer que la intención del Semanario ha sido decir que todos los románticos son unos locos: y $2^{\circ}$ suponer también que la intención del Semanario ha sido verter en ese artículo todo lo que sabía acerca del romanticismo. En cuanto a lo primero, nada menos que eso: porque dice que $R u y$ Blas de Víctor Hugo es malo, no se debe inferir que su intención ha sido decir que todos los románticos son unos locos. En cuanto a lo segundo, habría también igual fundamento para creer que en un artículo que tuviese por epígrafe, por ejemplo, "Chile", el autor había escrito todo lo que sabía de Chile. En su crítica ha cometido todavía otro error: el suponer que Thiers, Guizot, Gibbon, Niebuhr y otros cuantos historiadores que nos cita son escritores románticos. Porque si el romanticismo consiste en no sujetarse a regla alguna, no vemos qué tengan de románticos los escritores dichos; entonces podría decirse también que Thucidides, Nepote, Tito Livio $y$ todos los historiadores de la antigüedad eran románticos; lo cual sería un disparate.

(Gaceta del Comercio, Valparaíso, núm. 157, 8 de agosto de 1842).

John-Kenneth LesLIE, Northwestern University.

NOTAS

1 La polémica del romanticismo en 1842 (V. F. López, D. F. Sarmiento, S. Sanfuentes). Buenos Aires, Editorial Americalee, (1942). (Colección Tiempo de América).

2 "Panorama y significación del movimiento literario de 1842 ", en 1842, por Norberto Pinilla, Manuel Rojas y Tomás Lago, Santiago de Chile, Ediciones de la Universidad de Chile, 1942, págs. 7-38. La generación chilena de 1842, Santiago de Chile, 1943. 
3 Véase mi libro sobre el dramaturgo argentino Ventura de la Vega y el teatro español del periodo romántico: Ventuta de la Vega and the Spanish Theatte (1820-1865), Princeton, 1940.

4 El primer artículo de López, Clasicismo y romanticismo, quedó inconcluso. E1 segundo, intitulado Consideraciones sobre el tomanticismo, fué publicado en seis partes.

5 Obras de D. F. Sarmiento, 1, Reimpresión, París, Belin Hermanos, Editores, 1909, págs. 289.331. Tengo para mí que Pinilla acertó al omitir de su recopilación el primero de los artículos de esta colección, El prospecto del Semanario de Santiago, pues en verdad no tiene aue ver nada con la cuestión del tomanticismo. Sin embargo, está en su debido lugar en las Obras, porque es de carácter polémico, sirve de introducción a los demás artículos y pone de manifiesto algunos de los motivos que tenía Sarmiento para lanzarse de una manera tan vehemente en medio de una controversia tan amarga.

6 Sarmiento, Obras, I, pág. 296; Pinilla, Polémica, pág. 78.

7 Sanfuentes, en el artículo citado a continuación, afirma que fué "la denominación que le daba (al teatro) en sus románticos carteles un romántico compatriota vuestro". Parece que se refiere al actor argentino Juan Aurelio Casacuberta, quien había emigrado a Chile en 1841 y actuaba en el teatro de Santiago.

8 Semanario de Santiago, núm. 3 (28 de julio de 1842), pág. 24 : Pinilla, Polémica, pág. 119.

9 Véase mi artículo, "Problems Relating to Sarmiento's Atticulos criticos $i$ literatios", en Modern Language Notes, LXI (1946), págs. 289-299.

10 Sarmiento, Obras, I, págs. 305-309; Pinilla, Polémica, págs. 91-96.

11 Sarmiento, Obtas, I, pág. 295: Pinilla, Polémica, pág. 77.

12 Sarmiento, Obras, I, pág. 303; Pinilla, Polémica, pág. 88.

13 Sarmiento, Obras, I, pág. 304; Pinilla, Polémica, pág. 88.

14 Sarmiento, Obras, I, pág. 306; Pinilla, Polémica, pág. 93.

15 Pinilla, Polémica, pág. 38.

16 Sarmiento, Obras, I, pág. 295; Pinilla, Polémica, pág. 77.

17 Sarmiento, Obras, I, pág. 303; Pinilla, Polémica, pág. 88.

18 Sobre este particular, véase Ricardo Rojas, El profeta de la pampa. Buenos Aires, 1948, págs. 110-118.

19 Núm. 157 ( 8 de agosto de 1842), "Correspondencia", págs. 2-3. 